\title{
Study of five-dimensional potential-energy surfaces for actinide isotopes by the macroscopic-microscopic method
}

\author{
T.S. Fan ${ }^{\mathrm{a}}$, Z.M. Wang, X. Zhu, W.J. Zhu, and C.L. Zhong \\ State Key Laboratory of Nuclear Physics and Technology, Institute for Heavy ion Physics, School of Physics, Peking University, \\ Beijing 100871, P.R. China
}

\begin{abstract}
In this work, the nuclear potential-energy of the deformed nuclei as a function of shape coordinates is calculated in a five-dimensional (5D) parameter space of the axially symmetric generalized Lawrence shapes, on the basis of the macroscopic-microscopic method. The liquid-drop part of the nuclear energy is calculated according to the Myers-Swiatecki model and the Lublin-Strasbourg-drop (LSD) formula. The Woods-Saxon and the folded-Yukawa potentials for deformed nuclei are used for the shell and pairing corrections of the Strutinsky-type. The pairing corrections are calculated at zero temperature, T, related to the excitation energy. The eigenvalues of Hamiltonians for protons and neutrons are found by expanding the eigen-functions in terms of harmonic-oscillator wave functions of a spheroid. Then the BCS pair is applied on the smeared-out single-particle spectrum. By comparing the results obtained by different models, the most favorable combination of the macroscopic-microscopic model is known as the LSD formula with the foldedYukawa potential.

Potential-energy landscapes for actinide isotopes are investigated based on a grid of more than 4,000,000 deformation points and the heights of static fission barriers are obtained in terms of a double-humped structure on the full 5D parameter space. In order to locate the ground state shapes, saddle points, scission points and optimal fission path on the calculated 5D potential-energy surface, the falling rain algorithm and immersion method are designed and implemented. The comparison of our results with available experimental data and others' theoretical results confirms the reliability of our calculations.
\end{abstract}

\section{Introduction}

To reveal the characteristic observables of the fission process, one must calculate a multi-dimensional potentialenergy surface that guides the nuclear shape evolution from the ground state to the scission point. It is generally accepted that the macroscopic-microscopic (MM) method, which has its origins in early works of Strutinsky $[1,2]$, is a powerful tool in the large-scale calculation of nuclear potential energies. A series of recent investigations performed by Möller et al. showed the most important progress in understanding of fission mechanism with MM approach, in which they calculated the fission barrier heights for 1585 nuclei from $Z=78$ to $Z=125$ [3].

The macroscopic model stemmed from the concept of the charged liquid drop can be explained as the simplified form of leptodermous expansion [4] of the energy-density functional. Over the years various important new models with some new corrections have been proposed to improve its accuracy, such as the Myers-Swiatecki liquid-drop (MS-LD) model [5], the finite-range liquid-drop model [6] and the Lublin-Strasbourg drop (LSD) model [7], and so on. The deformed Woods-Saxon [8] and the folded-Yukawa formula of single-particle potential [9] are widely used in the MM calculations. The parametrization of the nuclear shapes should accurately describe the shape evolution from the ground state to the scission configuration in the multidimensional deformation space.

a e-mail: tsfan@pku.edu.cn
Several popular parameterizations are available such as the Cassinian ovals [10], the Funny-Hills parameterization [11], the three connected quadratic surfaces [12] and the generalized Lawrence shapes [13].

In this report, we present calculations of potentialenergy landscape of actinide isotopes in a five-dimensional (5D) deformation parameter space of the axially symmetric generalized Lawrence shapes, on the basis of the macroscopic-microscopic method. The liquid-drop part of the nuclear energy is calculated by using the Myers-Swiatecki model and the LSD formula [14], and the Woods-Saxon and the folded-Yukawa potentials of deformed nuclei is applied for the shell and pairing corrections of the Strutinsky-type. Two algorithms are presented to locate the ground state shape, saddle points, scission points and optimal fission path on the calculated $5 \mathrm{D}$ potential-energy surface. The comparison of our results with available experimental data and others' theoretical results confirms the reliability of our calculations.

\section{Macroscopic-microscopic theory}

The total potential energy consists of a smoothly varying liquid drop and a shell effect part calculated by Strutinsky procedure as follows:

$$
E=E_{\mathrm{mac}}+\delta E_{\mathrm{mic}},
$$

both depending on the nuclear deformation. The MyersSwiatecki liquid-drop model and the LSD formula for the 
liquid-drop part $E_{\mathrm{mac}}$, and the Woods-Saxon and the folded Yukawa potentials for the shell effect term $\delta E_{\text {mic }}$ were taken in this calculation, respectively.

\subsection{Macroscopic model}

\subsubsection{Myers-Swiatecki liquid drop model}

Myers-Swiatecki liquid-drop formula contains the deformation dependent surface energy and Coulumb energy terms

$$
\begin{aligned}
E_{\text {mac }}= & -a_{2}\left(1-\kappa I^{2}\right) A^{2 / 3} B_{\text {surf }}(\text { def }) \\
& +\frac{3}{5} \frac{e^{2}}{4 \pi \varepsilon_{0}} \frac{Z^{2}}{r_{0}^{\mathrm{ch}} A^{1 / 3}} B_{\text {Coul }}(\text { def }),
\end{aligned}
$$

with the isospin asymmetry $I=(N-Z) / A$, and

$$
\begin{aligned}
& a_{2}=17.9439 \mathrm{MeV} \\
& \kappa=1.7826 \\
& r_{0}=1.2249 \mathrm{fm} .
\end{aligned}
$$

Functions $B_{\text {surf }}$ (def) and $B_{\text {Coul }}$ (def) of Eq. (2) are the dimensionless ratio of the surface and Coulumb energies of a deformed nucleus to that of the spherical one.

\subsection{2. $L S D$ formula}

The LSD model, as an improved version of the LDM, contains an explicit introduction of surface-curvature term, which makes it more suitable for fission study. The following formula of LSD model consists of the deformation dependent surface, the surface-curvature and the Coulomb energy terms

$$
\begin{aligned}
E_{\mathrm{mac}}= & b_{\text {surf }}\left(1-\kappa_{\text {surf }} I^{2}\right) A^{2 / 3} B_{\text {surf }}(\text { def }) \\
& +b_{\text {cur }}\left(1-\kappa_{\text {cur }} I^{2}\right) A^{1 / 3} B_{\text {cur }}(\text { def }) \\
& +\frac{3}{5} \frac{e^{2}}{4 \pi \varepsilon_{0}} \frac{Z^{2}}{r_{0}^{\text {ch }} A^{1 / 3}} B_{\text {Coul }}(\text { def })
\end{aligned}
$$

where

$$
\begin{aligned}
b_{\text {surf }} & =16.9707 \mathrm{MeV}, \\
\kappa_{\text {surf }} & =2.2938 \\
b_{\text {cur }} & =3.8602 \mathrm{MeV}, \\
\kappa_{\text {cur }} & =-2.3764 \\
r_{0} & =1.21725 \mathrm{fm} .
\end{aligned}
$$

Function $B_{\text {cur }}$ (def) of Eq. (3) is the dimensionless ratio of the surface energy of a deformed nucleus to that of the spherical ones. $B_{\text {surf }}($ def $)$ and $B_{\text {Coul }}($ def $)$ are the same with the definition of Myers-Swiatechi formula.

\subsection{Microscopic correction method}

The Hamiltonian of a nucleon is written by

$$
H=\frac{p^{2}}{2 m}+V+V_{\mathrm{SO}}+V_{\mathrm{C}},
$$

where $\boldsymbol{p}$ represents the vector operator of momentum and $m$ the mass of either a neutron or a proton. The first choice of spin-independent nuclear part of the potential is the Wood-Saxon potential as

$$
V_{1}(\rho, \zeta)=-\frac{V_{0}}{1+\exp \left[\left(L(\rho, \zeta)-R_{0}\right) / a\right]},
$$

where $V_{0}$ represents the well depth, $a$ is a range parameter, and $R_{0}$ the radius of spherical nucleus. The second choice of the spin-independent nuclear part is the folded-Yukawa potential

$$
V(\boldsymbol{r})=-\frac{V_{0}}{4 \pi a^{3}} \int_{V} \frac{e^{-\left|\boldsymbol{r}-\boldsymbol{r}^{\prime}\right| / a}}{\left|\boldsymbol{r}-\boldsymbol{r}^{\prime}\right| / a} \mathrm{~d} \boldsymbol{r}^{\prime}
$$

where $V_{0}$ is the well depth, and $a$ the range of the Yukawa folding function. The spin-orbit interaction is given by

$$
V_{\mathrm{SO}}(\boldsymbol{r})=-\lambda\left(\frac{\hbar}{2 m c}\right)^{2} \frac{\boldsymbol{\sigma} \cdot \nabla V(\boldsymbol{r}) \times \boldsymbol{p}}{\hbar},
$$

where $\lambda$ is the spin-orbit interaction strength, and $\sigma$ the Pauli spin matrices. The Coulomb potential part is defined by

$$
V_{\mathrm{C}}(\boldsymbol{r})=-\frac{3 Z e^{2}}{4 \pi R_{0}^{3}} \int_{V} \frac{\mathrm{d} \boldsymbol{r}^{\prime}}{\left|\boldsymbol{r}-\boldsymbol{r}^{\prime}\right|},
$$

where $R_{0}$ is the radius of the spherical nucleus. A detailed description of the parameters used in Eqs. (4)-(6) is given in Ref. [15].

Eigenvalues of Eq. (4) are evaluated by expanding the eigenfunctions in terms of harmonic-oscillator(HO) wave functions for a spheroid [1,7]. For each given nuclear surface shape, the frequencies $\omega_{\zeta}$ and $\omega_{\rho}$ of $\mathrm{HO}$ are determined by requiring the corresponding potential to simulate $V$ best within the nucleus [12].

The microscopic energy $\delta E_{\text {mic }}$ can be usually written as

$$
\delta E_{\text {mic }}=\delta E_{\text {shell }}+\delta E_{\text {pair }},
$$

where $\delta E_{\text {shell }}$ and $\delta E_{\text {pair }}$ are the shell correction energy and the energy correction due to the pairing correlations between nucleons, respectively. $\delta E_{\text {shell }}$ was evaluated from the computed single-particle spectra according to Strutinsky's smoothing method of sixth order with the smearing width $\gamma=8 \mathrm{MeV}$ [8]. The pairing correction energy $\delta E_{\text {pair }}$ was treated with BCS approximation.

\subsection{Shape parameterization}

The potential-energy surfaces are calculated in the generalized Lawrence shape parameterization. In cylindrical coordinates, the axially generalized Lawrence shape is defined as a power series expansion of $\rho^{2}$ with respect to $\zeta$ :

$$
\rho^{2}(\zeta)=\left(l^{2}-\zeta^{2}\right) \sum_{n=0}^{N} a_{n}(\zeta-z)^{n}
$$

where the semilength $l$ measures the elongation of the nucleus and the position on the neck $\mathrm{z}$ measures the mass asymmetry. Other three geometric parameters, including the neck radius $r$, the neck curvature $c$ and the center of mass position $s$, long with $l$ and $\mathrm{z}$, consist of the whole set of the shape parameters $[12,14]$. 
In the present calculations, we investigate the shapes that correspond to the following set:

$$
\begin{aligned}
l & =1.140 \ldots(0.035) \ldots 2.085 \\
r & =r_{0} \ldots(-0.02) \ldots r_{0}-0.38 \\
z & =0.00 \ldots(0.02) \ldots 0.46 \\
c & =c_{0} \ldots(0.09) \ldots c_{0}+1.53 \\
s & =0.0000 \ldots(-0.0175) \ldots-0.2975
\end{aligned}
$$

In the set, numbers in the parentheses specify the step with which the calculation is performed for a given variable. The starting values of $r$ and $c$ at a given $l$ are given by

$$
\begin{aligned}
& r_{0}=l^{-0.5}+0.05, \\
& c_{0}=-l^{-2.5}-0.5 .
\end{aligned}
$$

This choice of shape parameters finally yields more than 4,000,000 different shapes for each nucleus.

\section{Searching for fission barriers and results}

The immersion method introduced in Ref. [16] and later elaborated in Ref. [3] allows the unambiguous identification of the lowest saddle point between two local minima. A major drawback of this method is that it is rather time-consuming as it iterates over the grid quite many times to locate a saddle point.

The falling rain algorithm is presented here. Suppose a heavy rain pours over the entire investigated region, the optimal path between two arbitrary minima can be found by tracking the surface runoffs. This algorithm implemented in our study turns out to be much faster than the immersion method.

The double-humped structures of fission barriers for $\mathrm{U}$ and $\mathrm{Pu}$ isotopes are presented by the macroscopicmicroscopic calculations using the LSD model and the folded Yukawa single-particle potential in this work. As an example, Fig. 1 shows the calculated results for the inner barrier height $E_{A}$ and the outer barrier height $E_{B}$ for $U$ isotopes. As seen in Fig. 1, our calculations agree quite well with the experimental data (Maslov) in the outer barrier as the largest discrepancy being $1.4 \mathrm{MeV}$, while the inner barrier height show an underestimation of $1 \sim 2 \mathrm{MeV}$ on the whole. We think this inconsistency is acceptable for two reasons. First, the experimental barrier height is deduced from modeling measured fission exciting functions, and the PES is discrete, so the real fission barrier is probably not on the grid. Second, the primary (highest) barrier is always the most accurately measured one and plays a dominant role in estimating fission cross sections and fission half-lives. Our calculated primary barrier is always the outer one, while for the experimental data it is the outer one for $\mathrm{A} \leqslant 236$ and the inner one for $\mathrm{A}>$ 236. Therefore the discrepancy between calculated values and experimental data drops in the $\mathrm{A}>236$ region if the primary barrier is compared other than the outer one.

\section{Conclusions}

Potential energy surfaces of uranium isotopes have been investigated in a five-dimensional deformation space of the generalized Lawrence shapes. The macroscopicmicroscopic model employed in our calculations is
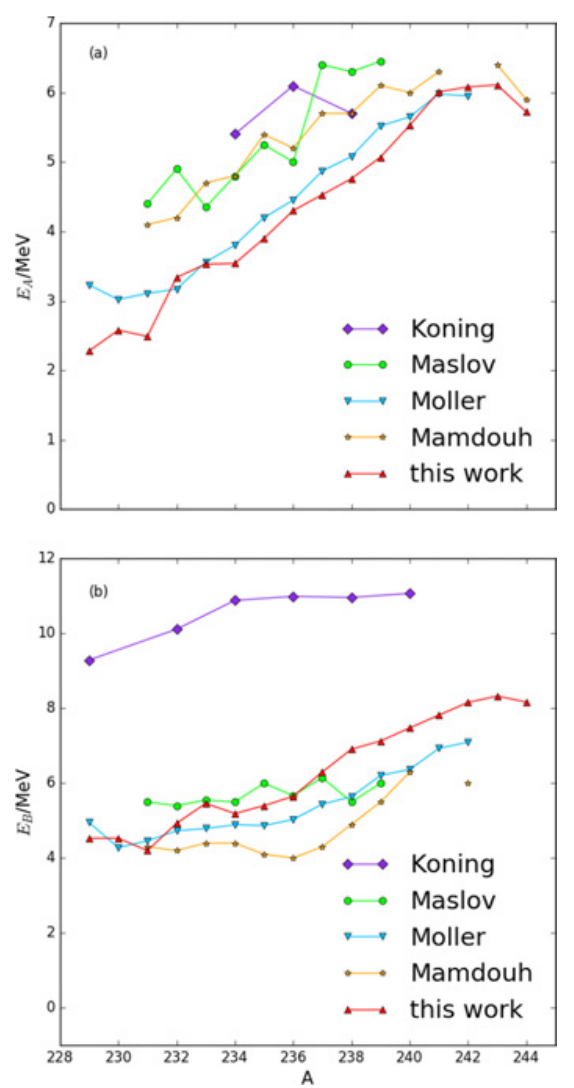

Figure 1. Comparison of our calculated inner barrier height $E_{A}$ (a) and outer barrier height $E_{B}$ (b) for $\mathrm{U}$ isotopes with experimental data and other theoretical efforts. The experimental data recommended by Maslov are taken from the Reference Input Parameter Library (RIPL-3) [17,18]. Möller's data are taken from Ref. [3]. Koning's values are obtained from both Ref. [19] and the nuclear structure database of nuclear reaction code TALYS 1.4 [20]. Mamdouh's ETFSI results are taken from Ref. [21].

finally based on the LSD model and the folded Yukawa single-particle potential. In order to resolve the complexity of searching for the optimal fission path on a multidimensional surface, we design and implement a new fast algorithm. The comparison of our results with available experimental data and others' theoretical results confirms the reliability of our calculations. This work would provide the basis for fully dynamical calculations of adequate precision in five shape parameters description of the fission process. Studies directed towards this goal are presently under way.

This work was supported by the National Natural Science Foundation of China (No. 91226102), the National Magnetic Confinement Fusion Science Program of China (Nos. 2013GB106004 and 2012GB101003), and the National Key Research and Development Program of China (No. SQ2016YFZG020067).

\section{References}

[1] Strutinsky V.M., Nucl. Phys. A 95, 420 (1967)

[2] Brack M., Pauli H.C., Nucl. Phys. A 207, 401 (1973)

[3] Möller P., Sierk A.J., Ichikawa T., et al. Phys. Rev. C 79, 064304 (2009)

[4] Myers W.D., Swiatecki W., J. Ann. Phys. (N.Y.) 84, 186 (1974) 
[5] Myers W.D., Swiatecki W., J. Nucl. Phys. 81, 1 (1966)

[6] Möller P., Myers W.D., Swiatecki W.J. et al., At. Data Nucl. Data Tab. 39, 225 (1988)

[7] Pomorski K, Dudek J., Phys. Rev. C 67, 044316 (2003)

[8] Damgaard J., Pauli H., Pashkevich V. et al., Nucl. Phys. A 135, 432 (1969)

[9] Bolsterli M., Fiset E.O., Nix J.R. et al. Phys. Rev. C 5, 1050 (1972)

[10] Pashkevich V.V. Nucl. Phys. A 169, 275 (1971)

[11] Brack M., Damgaard J., Jensen A.S. et al., Rev. Mod. Phys. 44, 320 (1972)

[12] Nix J.R. Nucl. Phys. A 130, 241 (1969)
[13] Brosa U., Grossmann S., Müller A., Phys. Rep. 197, 167 (1990)

[14] Zhong C.L., Fan T.S., Commun. Theor. Phys. 62, 405 (2014)

[15] Möller P., Nix J.R., Myers W.D. et al., At. Data Nucl. Data Tab. 59, 185 (1995)

[16] Olofsson H., Bengtsson R., Möller P. Nucl. Phys. A 784, 104 (2007)

[17] https://www-nds.iaea.org/RIPL-3/

[18] Capote R., et al., Nucl. Data Sheets. 110, 3107 (2001)

[19] Duijvestijn M.C., Koning A.J., Hambsch F., J. Phys. Rev. C 64, 014607 (2001)

[20] http://www.talys.eu/

[21] Mamdouh A., Pearson J.M., Rayet M., et al. Nucl. Phys. A 644, 389 (1998) 